\title{
Angiocentric invasion by lentigo maligna melanoma
}

\author{
S Kerr, J J Going
}

Department of
Pathology, Glasgow
Royal Infirmary,
Castle Street, Glasgow
G4 0SF
S Kerr
J J Going
Correspondence to:
Dr J J Going
Accepted for publication
18 August 1993

\begin{abstract}
A case of angiocentric invasion by a lentigo maligna melanoma is reported. Pericapillary cuffing and permeative intimal expansion of veins by melanoma without luminal tumour cell emboli, thrombosis, or vascular destruction were associated with a lentigo maligna melanoma on the face of a 93 year old woman. This unusual infiltrative pattern suggests a specific interaction between melanoma cells and perivascular connective tissue elements, and may imply expression by
\end{abstract}

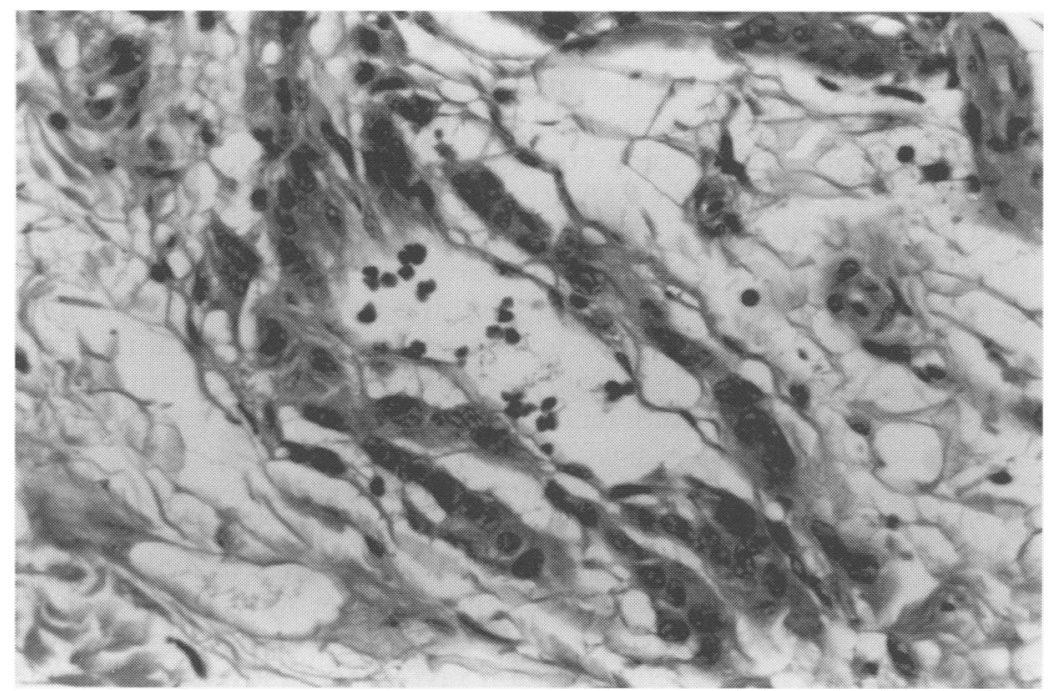

Figure 1 Pericapillary infiltration by melanoma cells.

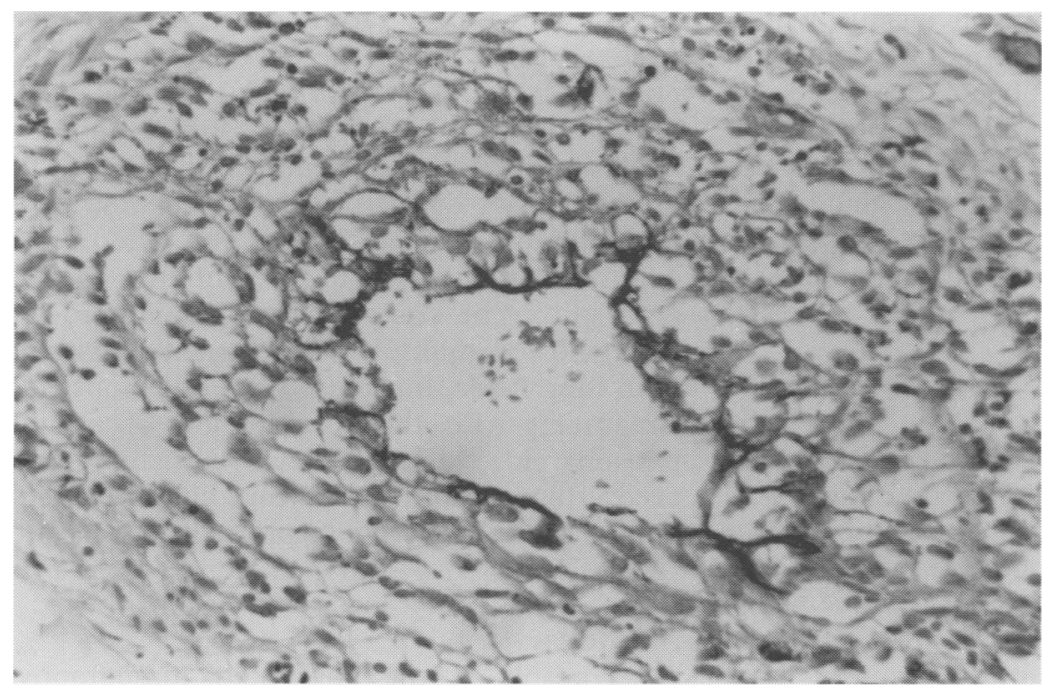

Figure 2 Immunohistochemistry for factor VIII related antigen showing preservation of venous endothelium. tumour cells of receptors for molecules in the intima of blood vessels and in pericapillary stroma.

(F Clin Pathol 1994;47:183-184)

Vascular infiltration by malignant tumours is a commonplace observation in diagnostic histopathology. Tumour cells are mostly observed in the lumina of lymphatics and blood vessels; destruction of vessel walls may also occur. We describe a pattern of angiocentric infiltration by melanoma which is almost the inverse of the usual infiltrative pattern.

\section{Case report}

A lentigo maligna melanoma was excised from the face of a woman aged 93. The flat lentiginous component measured $35 \times$ $30 \mathrm{~mm}$; a raised pigmented papule measured $15 \times 12 \mathrm{~mm}$. Histological examination showed extensive lentigo maligna. The raised area was due to invasive lentigo maligna melanoma (Clark level 4; Breslow thickness $2 \cdot 1 \mathrm{~mm}$ ). Extensive perineural infiltration was also present, and there was striking cuffing of dermal capillaries by melanoma cells (fig 1), but tumour emboli were not identified in blood or lymphatic vascular lumina. Most remarkable was extensive infiltration and expansion of the intima of larger blood vessels (mostly veins) by melanoma. The endothelium was generally raised but preserved. Thrombosis was not observed in any of the many vessels so affected. Preservation of endothelium was confirmed by immunohistochemical staining for vascular markers (Q-Bend 10, factor VIII related antigen, and Ulex europaeus lectin binding) (fig 2). The tumour cells were uniformly positive for S100 protein.

\section{Discussion}

Angiocentric invasion by lymphoma is well recognised, ${ }^{12}$ but the pattern of invasion we describe seems to be most unusual. Two similar cases were reported in $1992 .{ }^{3}$ It seems that in these cases there may have been some special affinity between the malignant cells and the stromal environment adjacent to vascular endothelium. Perhaps this was caused by expression on the melanoma cells of receptors for an extra-cellular matrix component localised around capillaries and 
in the vascular intima. Another possible mechanism might be increased susceptibility of this compartment to matrix degradation, providing a favoured infiltrative route. Interestingly, the tumour we report also showed pronounced neurotropism; there may be a functional connection here, but as neurotropism is commonly seen on its own, there can hardly be just one mechanism operating to produce both infiltrative patterns. Stromal desmoplasia and pronounced elongation of melanocytes were absent in our case; therefore, it does not seem to belong to either of the specific subtypes, desmoplastic malignant melanoma or its neurotropic variant. ${ }^{45}$

1 Colby TV, Carrington CB. Pulmonary lymphomas simulating lymphomatoid granulomatosis. Am ₹ Surg Pathol 1982;6:19-32.

2 Katzenstein A, Carrington CB, Liebow AA. Lymphomatoid granulomatosis: a clinico-pathologic study of 152 cases. Cancer 1979;43:360-73.

3 Moreno A, Espanol I, Ramogosa V. Angiotropic malignant melanoma. Report of two cases. $\mathcal{F}$ Cutan Pathol nant me:3250ma.

4 Bruijn JA, Mihm MC, Barnhill RL. Desmoplastic melanoma. Histopathology 1992;20:197-205.

5 Mack EE, Gomez EC. Neurotropic melanoma. A case report and review of the literature. $\mathcal{f}$ Neuro-oncol 1992; 13:165-71.

\title{
Signet-ring cell lymphoma of bone marrow
}

\author{
D C Talbot, J H Davies, K A Maclennan, I E Smith
}

\begin{abstract}
A case of signet-ring cell lymphoma affecting bone marrow is reported. The tumour presented as multiple lytic lesions in the lumbosacral spine. A bone biopsy specimen showed the typical appearances of signet-ring cell lymphoma, and the cells stained positively with antiserum to CD20, though neither immunoglobulin light or heavy chains could be shown within the vacuoles. The patient subsequently responded to chemotherapy.
\end{abstract}

( Clin Pathol 1994;47:184-186)

Signet-ring lymphomas are a rare form of non-Hodgkin's lymphoma first described by Kim et al in 1978. ${ }^{1}$ They have a characteristic morphological appearance as a result of large vacuolated or eosinophilic inclusions which displace the nucleus to the periphery. Signetring lymphomas have been described in lymph nodes and in a variety of other organs including the orbit, ${ }^{2}$ skin $^{3}$ central nervous system, ${ }^{4}$ tonsils, ${ }^{5}$ thyroid, ${ }^{6}$ salivary gland ${ }^{7}$ and stomach. ${ }^{8}$ We report here the first case (as far as is known) of signet-ring cell lymphoma affecting bone marrow.

\section{Case report}

A 56 year old woman was referred for a second opinion regarding the further investigation and management of multiple lytic lesions of the lumbosacral spine. She had initially presented in Portugal 10 months earlier with a history of back pain which was treated with simple analgesia and physiotherapy. Her symptoms deteriorated, and three months later plain radiography showed a lytic lesion in the body of $\mathrm{Ll}$ causing partial collapse. A computed tomogram of the lumbosacral spine confirmed lytic lesions in L1, L5, and S1. The patient underwent surgical stabilisation and insertion of Harrington rods, with sub-laminary wires from D10 to $\mathrm{L} 2$, with a bone graft from the left ilium. A bone biopsy specimen at this time had shown necrotic tissue only, and she was given quadruple antituberculous chemotherapy on an empirical basis.

At the time of referral, three months after the operation, she had considerable back pain, limiting her mobility. The only abnormal physical sign of note was reduced dorsiflexion of the left halux. Her erythrocyte sedimentation rate was $7 /$ first hour and a full peripheral blood count was normal, including a differential white cell count. Liver function tests, including alkaline phosphatase and serum calcium were normal, as was serum electrophoresis. Urinary Bence-Jones proteins were absent. Plain radiography and computed tomography confirmed extensive lytic deposits in the lumbo-sacral spine. A bone marrow aspirate and trephine biopsy specimen were not diagnostic and so a computed tomography guided biopsy of the lytic deposits was performed. A computed tomogram of the chest as part of the staging procedure showed multiple small opacities within both lung fields, but these were not biopsied. Treatment with the combination of cyclophosphamide, doxorubinin, vincristine and prednisolone resulted in symptomatic benefit and an objective partial response.

\section{Methods}

As a result of the computed tomography guided biopsy, several pieces of bone were received, the largest about $2 \mathrm{~cm}$ in diameter. 